1 Pronounced uptake and metabolism of organic substrates by diatoms revealed by pulse-

3

\title{
labeling metabolomics
}

Authors: Nils Meyer ${ }^{1}$, Aljoscha Rydzyk ${ }^{1}$, Georg Pohnert ${ }^{1,2 *}$

${ }^{1}$ Institute for Inorganic and Analytical Chemistry, Bioorganic Analytics, Friedrich Schiller University Jena, Lessingstrasse 8, D-07743 Jena, Germany

${ }^{2}$ Max Planck Institute for Chemical Ecology, Hans Knöll Str. 8, D-07745 Jena, Germany

*corresponding author: georg.pohnert@uni-jena.de

Classification: Biological Sciences, Microbiology, Ecology

Key Words: Phytoplankton Interactions, Diatoms, absorbotrophy, mixotrophy, mass spectrometry

\begin{abstract}
Diatoms contribute as a dominant group of microalgae to approximately $20 \%$ of the global carbon fixation. In the plankton, these photosynthetic algae are exposed to a plethora of metabolites, especially when competing algae are lysed. It is well established that diatoms can take up specific metabolites, such as vitamins, amino acids as nitrogen source, or dimethylsulfoniopropoionate to compensate for changes in water salinity. It is, however, unclear to which extent diatoms take up other organic resources and if these are incorporated into the cell's metabolism. Here, we ask about the general scope of uptake of metabolites from competitors. Using labeled metabolites released during lysis of algae grown under a ${ }^{13} \mathrm{CO}_{2}$ atmosphere, we show that the cosmopolitan diatom Chaetoceros didymus takes up organic substrates with little bias and remarkable efficiency. The newly developed pulse label/ mass spectrometry metabolomics approach reveals that polarity and molecular weight has no detectable influence on uptake efficiency. We also reveal that the taken-up pool of metabolites is partly maintained unaltered within the cells but is also subject to catabolic and anabolic transformation. One of the most dominant phytoplankton groups is thus substantially competing with other heterotrophs for organic material, suggesting that the observed absorbotrophy may substantially impact organic material fluxes in the oceans. Our findings call for the refinement of our understanding of competition in the plankton.
\end{abstract}

Significance This study demonstrates a remarkably universal uptake of organic substrates by diatoms. The extent to which one of the most dominant phytoplankton groups is competing for organic material in the plankton is documented by novel pulse labeling metabolomics studies. Our results show that uptake of organic material by the photosynthetic microalgae occurs with remarkably little bias. Taken-up metabolites are further transformed by the diatoms or directly incorporated into the algal metabolome. Our study calls for a re-consideration of organic material fluxes in the oceans. Also, our understanding of competition in the plankton will have to be refined. The broader implications for the cycling of resources in plankton communities are discussed within this work. 


\section{Introduction}

The traditional view on marine plankton distinguishes between phytoplankton as primary producers and zooplankton as consumers (1). However, many planktonic eukaryotic organisms have been recognized as mixotrophs, which combine autotrophic photosynthesis with organic matter uptake (2). Many microzooplankton grazers are mixotrophic and retain functional algal organelles or even algal endosymbionts. Also photosynthetically active organisms, such as phytoflagellates and dinoflagellates can engulf and consume prey organisms to acquire nutrients (3). As an additional strategy, the uptake of dissolved organic carbon termed absorbotrophic mixotrophy or osmotrophy can be observed in microalgae. This process seems to be ubiquitous but clearly less understood (4).

First investigations of absorbotrophic mixotrophy in plankton focused on algae growth under extreme darkness in the presence of organic substrates $(5,6)$. Administration and uptake studies of radiolabeled substrates deepened our mechanistic understanding. However, experiments were always limited to investigating one compound or a compound class, such as specific vitamins or amino acids. It is now clear that absorbotrophic mixotrphy is widely distributed among planktonic eukaryotic organisms (7). However, the experiments with single compounds under limiting conditions conducted so far represent an oversimplification and do not reflect the situation in nature, where a cell is exposed to structurally most diverse metabolites. Consequently, the importance of absorbotrophic mixotrophy for pelagic food webs and for element cycling remains elusive and we are still far from quantitatively deciphering the trophic modes of phytoplankton (8).

The availability of organic substrates for uptake will be highly variable. In the plankton mass occurrences of algae, so-called algal blooms can last over days to weeks before the population breaks down and is succeeded by other species that become dominant. Especially during the decay of such algal blooms, the surviving competitors will be exposed to the metabolites of the lysed algae. Also, lysis of specific phytoplankton members by pathogens, such as algicidal bacteria or viruses results in situations where surviving resistant cells are exposed to the metabolomes of the lysed species. $(9,10)$ It is entirely unclear if and how all these compounds contribute to the metabolism of the phytoplankton and the potential ecological importance of phytoplankton as consumers of organic material is thus still poorly understood (11-14).

As critical primary producers, diatoms were initially classified as autotrophs, but the uptake capability of specific organic molecules was early recognized $(14,15)$. These include 
glucose, small polar organic acids such as acetate, succinate, fumarate, malate and lactate, amino acids, dipeptides and dimethylsulfoniopropionate (DMSP) (16-23). Most experiments provided mechanistic insight but did not accurately reflect natural conditions, where the water in which the algae live harbors a diverse mixture of organic compounds. Under natural conditions, cells are exposed to these metabolites and also light is available to support photosynthesis. Thus, two competing mechanisms for carbon acquisition, uptake and heterotrophy will be active. Here we address absorbotrophic mixotrophy in diatoms under nonlimited conditions. The supply of nutrients and light in our study was non-limiting to allow efficient algal growth; organic metabolites were thus offered in addition to available inorganic sources.

We base our experimental setup on a well-investigated multi-partner interaction involving an algicidal bacterium and two cosmopolitan diatom species. Kordia algicida is a marine Flavobacterium that possesses algicidal activity leading to cell lysis of several microalgal species, including the diatom Skeletonema costatum $(24,25)$. Chaetoceros didymus, in contrast, is a naturally co-occurring diatom that is resistant against $K$. algicida. The impact of $K$. algicida was recently shown in field experiments where it induces a population shift in a natural phytoplankton community towards resistant algae (26). We hypothesize that during bacterial lysis of $S$. costatum, resistant species can benefit by taking up metabolites of the lysate in an absorbotrophic manner. Therefore, we exposed a culture of $C$. didymus to metabolites from a $50 \%$ diluted stationary culture of lysed $S$. costatum cells. We developed a novel analytical approach to test this hypothesis, including pulse labeling metabolomics and a novel non-discriminant data treatment routine. We show that uptake and metabolism of metabolites from the environment occur with remarkable efficiency in the resistant alga.

\section{Results}

Generation and evaluation of a labeled metabolome for uptake experiments

A complex medium containing the entire labeled metabolome of a diatom could be generated from ${ }^{13} \mathrm{C}$-labeled S. costatum. We grew $S$. costatum in a medium containing $\mathrm{Na}_{2}{ }_{2}^{13} \mathrm{CO}_{3}$ as a sole carbon source to obtain a labeled metabolome. With a repeated exchange of the medium, we reached up to $65 \%$ labeling in the algal metabolome. Mechanical lysis of the labeled culture and removing the cell debris gives an axenic medium, rich in organic metabolites (Suppl. Fig. 1). 
We hypothesized that the resistant $C$. didymus encounters these metabolites also in the field when algicidal bacteria lyse its competitors. To test this hypothesis, labeled $S$. costatum cultures were infected with $K$. algicida, which resulted in lysis of more than half of the diatom cells within six days. Ultrahigh-pressure liquid chromatography-high resolution mass spectrometry (UHPLC-HRMS) of metabolites extracted from the medium revealed similar metabolic profiles in mechanically lysed $S$. costatum cells and those lysed by the bacteria (Table $1)$.

Before incubation with $C$. didymus, the metabolites from lysed $S$. costatum were sterilized and diluted with a medium containing inorganic carbon with normal isotope distribution $\left(1.1 \%{ }^{13} \mathrm{C}\right.$, Suppl. Fig. 1). Thereby, we could ensure that ${ }^{13} \mathrm{C}$ labeled organic metabolites taken up from the medium can be distinguished from de novo synthesized compounds. Following the same procedure, a control medium was generated using S. costatum cells grown in a medium with natural isotope distribution that could be used to generate mass spectra for structure elucidation. Using fragmentation trees, database comparison and subsequent comparison to authentic standards, we identified several of the labeled metabolites in C. didymus (Table 1).

\section{Evaluation of uptake}

For uptake experiments, $C$. didymus was cultivated in a medium containing the sterilized labeled $S$. costatum metabolome or control medium for three days $(n=3)$. We selected the concentration of added metabolites to be equivalent to $50 \%$ of those released by a lysed stationary culture. Cells were then recovered by filtration and washed extensively. UHPLCHRMS analysis of the $C$. didymus metabolome, after being exposed to this medium under otherwise optimum growth conditions, revealed that the alga took up substantial amounts of labeled compounds from various metabolic classes. Quantitative analysis of labeling proved to be challenging in terms of chemoinformatic data treatment. Therefore, ions of the same metabolite, only differing in their number of incorporated ${ }^{13} \mathrm{C}$ were summarized in an isotopologue group using the software $\mathrm{X}^{13} \mathrm{CMS}$ (27). Of 5587 isotopologue groups (positive and negative ionization mode) detectable in the endo metabolome 2381 were significantly labeled with ${ }^{13} \mathrm{C}$ (Fig. 1A). After manual curation, 548 isotopologue groups were categorized according to their labeling pattern and analyzed regarding their retention time and mass to charge ratio. The degree of labeling was estimated using the probability mass function for Bernoulli trials (random experiments with precisely two possible outcomes, "success i.e. 
incorporation of ${ }^{13} \mathrm{C}$ " and "failure i.e. incorporation of ${ }^{12} \mathrm{C}$ ", in which the probability of success is the same every time the experiment is conducted):

$\mathrm{I}(\mathrm{m})=\frac{\mathrm{n} !}{\mathrm{m} !(\mathrm{n}-\mathrm{m}) !} \cdot \mathrm{p}^{\mathrm{m}} \cdot(1-\mathrm{p})^{\mathrm{n}-\mathrm{m}}$

The degree of labeling $\mathrm{p}$ for a metabolite with $\mathrm{n}$ carbon atoms is estimated from the intensities of a set of isotopologues $\mathrm{I}(\mathrm{m})$ with $\mathrm{m}{ }^{13} \mathrm{C}$ atoms (28).

\section{Classification of taken-up and processed metabolites}

We categorized the metabolites according to their degree of labeling (Fig. 1B, C). $73 \%$ of the isotopologue groups were lowly labeled with a degree of labeling $<5 \%$ indicative of compounds mainly synthesized de novo in C. didymus. The low degree of labeling that still exceeds the natural ${ }^{13} \mathrm{C}$ content of $1.1 \%$ can be explained by general utilization of taken-up metabolites in the metabolism: metabolites that are assimilated are catabolized to metabolic building blocks that are used together with the autotrophic metabolic pool and used for anabolism again. Seven percent of the isotopologue groups were highly labeled. They contained a degree of labeling similar to that of metabolites in the medium (ca. 65\%). The cellular content of these highly labeled metabolites taken up from lysed S. costatum shows that C. didymus assimilates metabolites that it does not (or only to a minimal extent) produce itself. Certain compounds with a high degree of labeling can be found in $C$. didymus but not in the mixotrophic medium. These could arise from the metabolic transformation of more complex metabolites released by $S$. costatum. $15 \%$ of the isotopologue groups were labeled in a mixed manner. In the mass spectrum both, signals from a lowly labeled share and a highly labeled share of the respective compound can be detected. This pattern can be explained by metabolites that are biosynthesized by $C$. didymus and also acquired from the medium. This compound class includes a wide range of natural products, from small charged molecules like choline and carnitine to non-polar lipids and fatty acids. A few signals (5\%) had a complex labeling pattern that Bernoulli statistics could not describe. These can be interpreted as compounds that result from metabolites taken up and further metabolized using the pool of de novo synthesized metabolites (Fig. 1B, C). For example, intermediate metabolic products like fatty acids can be utilized in the anabolism of more complex ones like lipids.

\section{Properties of taken up metabolites}

Labeled metabolites span a wide range of polarity from charged small molecules to non-polar fatty acids. Most labeled products are found in the non-polar region of the chromatogram (Fig. 1D, Tab. 1, Suppl. Fig. 2). The identified polar metabolites that are efficiently taken up include the amino acids leucine and/or isoleucine and several small charged molecules, namely glycine 
betaine, $\beta$-alanine betaine, carnitine (58\% of total cellular carnitine is labeled), and choline. Also, many fatty acids and lipids are taken up, including oleic acid, 5Z,8Z,11Z,14Z,17Zeicosapentaenoic acid, 6Z,9Z,12Z-hexadecatrienoic acid, 6Z,9Z,12Z,15-hexadecatetraenoic acid, 2-hydroxytetradecanoic acid and isomers of linoleic and arachidonic acid as well as the lipid 1-oleoyl-sn-glycero-3-phosphocholine.

Metabolites that were taken up also span a wide $\mathrm{m} / \mathrm{z}$ range, with a maximum between $\mathrm{m} / \mathrm{z} 200$ and $m / z 700$ (Fig. 1D, Tab. 1, Suppl. Fig. 2). There is thus relatively little size discrimination for the uptake.

\section{Analysis of selected metabolites}

Detailed analyses of the isotopic pattern enabled us to determine the ratio of heterotrophic uptake to de novo biosynthesis and look for evidence of mixed strategies. For example, the isotopologues of $6 Z, 9 Z, 12 Z, 15$-hexadecatetraenoic acid (Fig. 2A) originate from two distinct pools (Fig. 2D), a lowly labeled pool from de novo biosynthesis and a highly labeled pool from uptake. Modeling with Bernoulli statistics showed that the de novo biosynthesis pool had a degree of labeling of $2.8 \%$, slightly higher than what would be expected from photosynthesis using exclusively natural inorganic carbon (Fig. 2B). The highly labeled pool has a very similar isotopologue distribution compared to the fatty acid from the lysed labeled alga (Fig. 2C). Modeling a mixture of these two pools showed that ca. $20 \%$ of the metabolite in the algae results from uptake, while $80 \%$ are synthesized de novo. This complementation of de novo synthesized products with externally available metabolites is observed for several metabolites with variable proportions of the two sources from only a few percent to nearly $80 \%$ of the metabolite acquired by uptake (Table 1$)$.

The isotopologue patterns of metabolites that can be explained by an uptake of resources from the medium followed by transformations within the cell using the pool of de novo synthesized precursors are more complex. They do not follow the Bernoulli statistics since different resources can be utilized in different relative amounts. An example is the lysophosphatidylcholine shown in Fig. 3A. The isotope pattern of the lipid (Fig. 3B) cannot be interpreted with the model described above, but tandem MS experiments allow to dissecting the lipid. This reveals a unique labeling pattern for those parts of the molecule that are derived from different biosynthetic pathways. The isotopologue pattern of oleic acid in the lysophosphatidylcholine (Fig. 3C) can also not be fitted with a Bernoulli statistic. The fatty acid is thus assembled using resources that were taken up as well as de novo produced. This labeling pattern in the fatty acid moiety is also observed in free oleic acid and 11Z-eicosenoic acid. Lipid 
assembly thus does not discriminate between acquired and de novo synthesized resources. In contrast, the choline fragment detected in the same substance shows that most choline is highly labeled and therefore taken up (Fig. 3D). The glycerol moiety of the molecule is not giving charged fragments, but its labeling could be established indirectly. Therefore, we conducted fragmentation of the $\mathrm{M}+8$ isotopologue of the lysophosphatidylcholine. The MS/MS of this ion gave rise to an oleic acid fragment with isotopologues containing down to zero ${ }^{13} \mathrm{C}$ (Fig. 3E). The remaining eight carbon atoms in the uncharged C8-fragment have thus to be labeled in different degrees. The fragments can thus only be derived from a precursor with labeled, thus acquired glycerol.

These exemplarily discussed mass spectra stand for several hundreds of labeled peaks in the chromatogram of the metabolome of exposed C. didymus (see Fig. 1 and Supporting information). Using the combined results, we can now draw a picture of the absorbotrophic metabolism in C. didymus (Fig. 4). The uptake introduced here for C. didymus is not limited to this one species but broader distributed in diatoms. When the diatom Thalassiosira weissflogii was raised in the labeled medium as described for $C$. didymus, labeling patterns similar to the ones described above were detected (data not shown).

\section{Discussion}

\section{${ }^{13}$ C labeling allows tracing of metabolic shuttling between microalgae}

In order to study potential absorbotrophic mixotrophy in the diatom $C$. didymus a medium rich in ${ }^{13} \mathrm{C}$ labeled organic metabolites was prepared by mechanical lysis of a globally labeled $S$. costatum culture and removal of the cell debris by filtration. The resulting metabolome enriched medium showed a similar (but labeled) metabolic profile as the medium of an infection experiment where the lytic bacterium $K$. algicida lysed $S$. costatum. We thus conclude that $K$. algicida is a sloppy feeder not utilizing the entire algal metabolome but instead leaving substantial organic resources that it does not require or recover in the water. Surviving algae, such as the resistant competitor $C$. didymus will be exposed to these metabolites. Earlier, we observed that such exposure to metabolites released from lysed algae supports the growth of $C$. didymus if administered at low concentrations. However, no information about the underlying mechanism was available (29). The ability to take up released organic molecules may counterbalance the metabolic costs to maintain resistance mechanisms and would be highly advantageous, for example, during the collapse of a competing algal bloom as it was simulated in this study $(10,30)$. If the metabolic uptake also compensates in phases of very dilute phytoplankton abundance would have to be verified in follow-up studies. We have, however, 
no indication from the analysis of labeling that there would be a bias for the uptake of higher concentrated metabolites.

We reached up to $65 \%$ labeling in the $S$. costatum metabolome using repeated exchange of medium containing $\mathrm{Na}_{2}{ }^{13} \mathrm{CO}_{3}$ as an exclusive carbon source. Analysis of the C. didymus endometabolome after being exposed to the labeled metabolome of $S$. costatum for 3 days under otherwise optimum growth conditions revealed that the alga took up substantial amounts of labeled compounds from various metabolic classes. Given that analysis of every single isotopologue of a labeled metabolite would generate highly complex and partially redundant information, we reverted to a statistical treatment assuming that labeling results in an isotopologue distribution that can be described with a Bernoulli statistic. Indeed, this data treatment allowed to match mass spectral patterns of labeled metabolites to predicted spectra that would result from specific degrees of labeling. Thereby the average $65 \%$ labeling of the metabolome could be determined.

Labeling patterns of metabolites in C. didymus after exposure to ${ }^{13}$ labeled $S$. costatum medium

Analysis of the metabolome of the resistant alga $C$. didymus after exposure to ${ }^{13} \mathrm{C}$ labeled S. costatum revealed unlabeled metabolites, metabolites with unaltered full labeling and those with more complex mass spectra that could be assigned to different metabolic processing (Fig. 1). The methodology described here thus not only shows the uptake of one metabolite, but allows simultaneous quantification of the uptake and analysis of the metabolic fate of all taken up metabolites in the receiving alga. This is a valuable expansion of the classical fluxomics approach, where feeding of one single labeled metabolite to heterotrophs can only reveal its uptake and metabolism. Our experimental approach reflects the situation in the plakton with highly complex microbial communities and complex exometabolomes (31). It allows evaluation of the uptake capacity and incorporation of the taken-up metabolites under ecologically relevant conditions.

\section{General patterns in the uptake of organic metabolites by the receiving diatom $C$. didymus}

Detailed analysis of the mass spectra allows to draw a picture of absorbotrophy in $C$. didymus (Fig. 4). More than a quarter of all detected metabolites in C. didymus were labeled to different degrees (Figure 1). Nearly $10 \%$ showed the identical labeling pattern as those in the ${ }^{13}$ C-labeled S. costatum metabolome. These metabolites are not (or only to a minor extent) synthesized by the receiver but taken up and maintained in the cells. The major part of takenup metabolites showed mixed labeling, indicative for the uptake of a metabolite that is also synthesized by the receiver. This includes a wide range of natural products from small charged 
molecules like choline and carnitine to non-polar lipids and fatty acids. Metabolites acquired from the outside water can thus be metabolized in the same way as the de novo produced compounds, indicating that no compartmentation of assimilated material occurs. Instead, the autotrophic and the phototrophic pool are used for anabolism and catabolism. For example, the presence of complex labeling patterns can be explained by the use of intermediate fatty acids in the anabolism of more complex lipids. The complex isotopologue pattern of oleic acid and 11Z-eiosenoic acid, for example, might reflect a dynamic system with rounds of beta oxidation releasing labeled acetate and subsequent fatty acid re-assembly from this labeled and the own unlabeled acetate pool. Since the isotopologues of these fatty acids are not Bernoulli-distributed the relative amount of labeled acetate in the total acetate pool used for fatty acid biosynthesis might vary over time.

\section{Utilization of assimilated metabolites}

The fact that uptake has no apparent preference for nitrogen containing metabolites contradicts the assumption that diatoms use specific heterotrophic mechanisms to acquire reduced nitrogen (19). Notably, many metabolites with essential physiological functions are thus not exclusively produced de novo but taken up in high proportions. Control of physiological concentrations will thus have to include biosynthesis, catabolism as well as uptake. A universal uptake could be highly advantageous in the event of a lysis of a competing algal bloom (10, 30). But even under regular conditions in plankton, algae might encounter metabolites released by other members of the phytoplankton that they can take up and benefit from (32).

The observation of universal uptake of metabolites requires re-thinking of the interpretation of incubation studies with single labeled substrates. It is well documented that specific primary metabolites can be taken up by the cells under limiting conditions. Thus, under darkness, uptake of organic acids like lactate and malate was observed (21). Amino acid assimilation after peptide lysis was interpreted as nitrogen uptake mechanism (19) and uptake of the essential metabolite dimethylsulfoniopropionate (DMSP) was discussed as a way to compensate for the lack of own DMSP biosynthetic capabilities (23). We now expand this view and introduce that diatoms can take up a structural variety of metabolites released from competitors even under optimized growth conditions. Thus no specific compensation mechanisms but rather universal complementation of the own metabolome occurs but rather an universal supply with potentially valuable metabolites. This unspecific uptake of metabolites over a wide range of polarities and masses newly defines diatoms as a general sink of organic carbon in the sea. 
Of the multiple labeled metabolites, two groups will be exemplarily highlighted here, polar nitrogen containing metabolites and fatty acids. Polar metabolites that are taken up include glycine betaine, $\beta$-alanine betaine, carnitine and choline, with diverse physiological functions. Glycine betaine is a known osmolyte and uptake in diatoms has already been described under $\mathrm{N}$-limited conditions $(33,34)$. Choline is a biosynthetic precursor of glycine betaine (35) and important as structural element in phospholipids (11). It has been found as a free form and in lysophosphatidylcholine in this study (Table 1). However, the labeling pattern of the choline fragment of lysophosphatidylcholine differs from the one of the free form (Suppl. Fig. 3), indicating different origins. $\beta$-alanine betaine is a known osmoprotectant in plants and is biosynthesized via the methylation of $\beta$-alanine, a building block of coenzyme A (36). It has also been found in marine algae (37). Carnitine is a central metabolite in energy metabolism of all eukaryotic cells. It plays an essential role for the transport of fatty acids across the mitochondrial membrane. The utilization of carnitine by diatoms has been reported previously by measuring oxidation rates in a biofilm-forming freshwater diatom (38). The presence of labeled $N$-methyl groups (Suppl. Fig. 4) excludes lysine degradation (39) as a metabolic origin. The high proportion of heterotrophically acquired carnitine and the presence of many fatty acids among the taken-up metabolites is striking. It suggests a highly active transport mechanism for these high-energy fatty acids and the $N$-containing carnitine. It would be interesting to investigate whether an acylcarnitine-type transport system might facilitate the uptake of fatty acids across the cellular membrane. The presence of a protein homologous to a class I carnitine/acylcarnitine translocase in a diatom cell wall proteome supports this hypothesis (40). Several labeled fatty acids and derivatives have also been identified (Table 1). Eicosapentaenoic acid is one of the dominant fatty acids in diatoms and precursor of many bioactive oxylipins (11, 41-43). 6Z,9Z,12Z-hexadecatrienoic acid is the precursor of octadienal, an allelopathic polyunsaturated aldehyde (44). Also, oleic acid, 11Z-eicosenoic acid, and 6Z,9Z,12Z,15hexadecatetraenoic acid are common in diatoms (43, 45-48). Two other C18- and C20 polyunsaturated fatty acids were identified according to retention time and mass spectra. Comparison with synthetic standards showed that these fatty acids were isomers of linoleic and arachidonic acid. Fatty acids and their derivatives have multiple physiological functions. The fact that they are present in labeled and unlabeled forms in after the incubation experiments indicates an unbiased utilization of de novo and assimilated compounds. This might serve as a mechanism to avoid costly biosynthesis of fatty acids, which is supported by the observation that the fatty acids are not only internalized or adsorbed to lipidic structures due to their physicochemical properties but rather incorporated into the primary metabolism of the diatom. 
The broad range of polarity and molecular weight of assimilated compounds raises the question of the uptake mechanism. Candidate systems for fatty acid shuttling include specific transporters and genome data shows homolog candidate sequences $(40,49)$. But indeed, also unspecific absorption and incorporation mechanisms that do not require transporters will most likely be involved. Nevertheless, the question of how diatoms acquire exogenous metabolites is still open.

\section{Concluding remarks}

The diatom $C$. didymus takes up and incorporates metabolites from lysed competitors with surprising little bias. The same is true for another model diatom $T$. weissflogii, which might suggest a rather universal mechanism. The multitude of cellular functions in which these metabolites are involved suggests that uptake complements the internal metabolic pool resulting from many different biosynthetic pathways.

The universal absorbotrophic lifestyle of one of the most abundant algal classes in the oceans substantially changes our view of the metabolic shuttling in phytoplankton communities. These algae take up not only a few highly polar metabolites for e.g. nitrogen supply. They rather complement their metabolism quite universally with resources from the surrounding seawater. This process occurs even under illumination and is not related to the complementation of lacking photosynthate in the dark. Thus, in addition to bacteria, diatoms compete for the dissolved organic carbon in the plankton $(50,51)$. Our study has consequences for element cycling in the oceans and ecosystem dynamics that will have to be addressed in the future.

\section{Experimental design}

Diatoms were grown under optimum conditions and exposed to a labeled metabolome of a competitor. The diatoms were extracted using optimized protocols for metabolomics sampling and extracts were analysed by liquid chromatography mass spectrometry to evaluate the uptake of labeled metabolites.

\section{Algal culturing}

C. didymus was isolated by W. Kooistra from the Gulf of Naples (Stazione Zoologica Anton Dohrn, Naples, Italy) and $S$. costatum was obtained from the Roscoff Culture Collection (Roscoff, France). Both algae were cultivated in batch culture using artificial seawater medium(52) in $50 \mathrm{~mL}$ Greiner Bio-One cell culture flasks at $11-13^{\circ} \mathrm{C}$ under a $14: 10 \mathrm{~h}$ light : 
dark regime with an illumination of 20-25 $\mu \mathrm{mol}$ photons $\mathrm{m}^{-2} \mathrm{~s}^{-1}$. Development of cultures was followed by in-vivo Chl $a$ fluorescence using a Mithras LB 940 plate reader (excitation $430 \mathrm{~nm}$, emission $665 \mathrm{~nm}$ ).

\section{Global ${ }^{13}$ C-labeling}

For global ${ }^{13} \mathrm{C}$-labeling of $S$. costatum we used autoclaved artificial seawater medium that was prepared without addition of $\mathrm{NaHCO}_{3}$. An aliquot of this medium was utilized to dissolve $\mathrm{NaH}^{13} \mathrm{CO}_{3}$ (98 atom \%, Sigma-Aldrich, Munich, Germany). This solution, containing sufficient $\mathrm{NaH}^{13} \mathrm{CO}_{3}$ to reach a final concentration of $2.38 \mathrm{mM}$, was sterile filtered $(0.2 \mu \mathrm{m}$ pore size, Sarstedt Filtropur S) and transferred back to the medium bottle. Tissue culture flasks were filled to the neck in order to minimize the area for $\mathrm{CO}_{2}$ exchange with the atmosphere and were inoculated with $<1 \%(\mathrm{v} / \mathrm{v})$ of a stationary S. costatum culture. After growing to stationary phase, an aliquot was taken and transferred to fresh ${ }^{13} \mathrm{C}$-enriched medium $(<1 \%(\mathrm{v} / \mathrm{v}))$. After two of these cycles a plateau in the degree of labeling (verified by mass spectrometry as described below) was reached and the cultures were used for further experiments.

\section{Bacterial culturing}

K. algicida (30) was cultivated on marine broth agar at $30^{\circ} \mathrm{C}$ for 2 days. The bacterial lawn was removed with a sterile cotton swab and re-suspended in algal culturing medium to an $\mathrm{OD}_{550}$ of 0.5 determined on a Genesys 10S UV-Vis spectrophotometer (Thermo Fisher Scientific, Waltham, MA, USA).

\section{Co-culturing experiment and extraction of released metabolites}

S. costatum cultures reared in ${ }^{12} \mathrm{C}$ or ${ }^{13} \mathrm{C}$ medium were co-cultured in triplicates (185 mL each) with the $K$. algicida added to a final $\mathrm{OD}_{550}$ of 0.01 . After 6 days the lysed cultures were gently filtered $(0.2 \mu \mathrm{m}$ pore size $)$ and the flow-through extracted for metabolome analysis as follows. Solid phase extraction cartridges (Chromabond easy, Macherey-Nagel, Düren, Germany) were equilibrated with $4 \mathrm{~mL}$ of methanol (Chromasolv ${ }^{\odot}$ Plus, Sigma-Aldrich, Munich, Germany) and $4 \mathrm{~mL}$ of water (Chromasolv ${ }^{\odot}$ Plus, Sigma-Aldrich, Munich, Germany) before the filtrate $(170 \mathrm{~mL})$ was applied using vacuum with a flow rate $<1 \mathrm{~L} \mathrm{~h}^{-1}$. The cartridge was washed with $4 \mathrm{~mL}$ water, air-dried and then extracted via gravity flow using $2 \mathrm{~mL}$ of methanol followed by $2 \mathrm{~mL}$ of methanol/tetrahydrofuran 1:1 (tetrahydrofuran HiPerSolv, VWR, Dresden, Germany). This extract was frozen until further chemical analysis. 


\section{Tests for mixotrophy}

Stationary cultures $(45 \mathrm{~mL})$ of $S$. costatum reared in ${ }^{12} \mathrm{C}$ or ${ }^{13} \mathrm{C}$ medium were centrifuged (500 $\mathrm{x} \mathrm{g}, 15 \mathrm{~min}, 10^{\circ} \mathrm{C}$ ) and washed three times by repeated addition of $45 \mathrm{~mL}$ of ${ }^{12} \mathrm{C}$ medium to the harvested pellets and centrifugation. After the third washing step the supernatant was collected as control medium (this was processed in parallel to the cells and later served as control for the natural ${ }^{13} \mathrm{CO}_{2}$ ) and the cell pellet was re-suspended in $45 \mathrm{~mL}{ }^{12} \mathrm{C}$ medium. To disrupt cells, the suspension was frozen at $-20^{\circ} \mathrm{C}$, thawed and treated in an ice-cold ultrasonic bath for $10 \mathrm{~min}$. The lysate was filtered (1.2 $\mu \mathrm{m}$ pore size, GF/C, Whatman, GE Healthcare, Little Chalfont, United Kingdom), acidified to $\mathrm{pH} \leq 1$ for sterilization using $30 \%$ hydrochloric acid, incubated at $0^{\circ} \mathrm{C}$ for $10 \mathrm{~min}$ and subsequently neutralized under sterile conditions using a saturated sodium hydroxide solution. The solutions were stored at $-20^{\circ} \mathrm{C}$ until use. After thawing, the solutions were diluted 1:1 with ${ }^{12} \mathrm{C}$ medium to yield the final medium for the investigation of mixotrophy $\left({ }^{12} \mathrm{C}\right.$ organic $\&{ }^{12} \mathrm{C}$ inorganic or ${ }^{13} \mathrm{C}$ organic $\&{ }^{12} \mathrm{C}$ inorganic $) .45 \mathrm{~mL}$ aliquots of both media were extracted as described above for exometabolomic analysis to determine the organic metabolites. For determination of mixotrophy $110 \mathrm{~mL}$ aliquots of these media were inoculated with $1 \%(\mathrm{v} / \mathrm{v})$ stationary $C$. didymus culture in triplicates and cultivated for 3 days. Directly after inoculation and after 3 days of cultivation, samples (45 mL each) for intra- and extracellular metabolomics were processed. Samples were filtered (1.2 $\mu \mathrm{m}, \mathrm{GF} / \mathrm{C}$, Whatman, GE Healthcare, Little Chalfont, United Kingdom) and the flow-through processed for exometabolomic analysis as described above (see co-culturing experiment). The cells were washed off the filter with an ice-cold freshly prepared mixture of methanol/ethanol/chloroform (1:3:1) (ethanol LiChroSolv ${ }^{\odot}$, Merck, Darmstadt, Germany; chloroform HiPerSolv ${ }^{\mathcal{C}}$, VWR, Dresden, Germany). Extracts were treated in an ultrasonic bath for $10 \mathrm{~min}$, centrifuged (30,000 $\mathrm{x} \mathrm{g}, 15 \mathrm{~min}, 4^{\circ} \mathrm{C}$ ) and the supernatant was stored at $-20^{\circ} \mathrm{C}$. As a control to prove that no $\mathrm{NaH}^{13} \mathrm{CO}_{3}$ enrichment was present in mixotrophic media, the third wash supernatant (see above) was used as medium to cultivate $C$. didymus and metabolites were extracted as described. A graphical representation of the experimental setup can be found in Suppl. Fig. 1.

\section{Analysis of exo- and endometabolomes with LC-MS}

Extracts from cells and media (see above) were dried in a nitrogen flow at room temperature and were resuspended in up to $200 \mu \mathrm{L}$ methanol. Metabolites were separated on an UltiMate 3000 UHPLC (Thermo Fisher Scientific, Waltham, MA, USA) equipped with an Accucore C18 column $(100 \times 2.1 \mathrm{~mm}, 2.6 \mu \mathrm{m})$ at $25^{\circ} \mathrm{C}$ using water with $2 \%$ acetonitrile and $0.1 \%$ formic acid (A) and pure acetonitrile (B) as mobile phase. The gradient was as follows: $100 \% \mathrm{~A}$ for 0.2 
min, linear gradient to $100 \% \mathrm{~B}$ in $7.8 \mathrm{~min}, 100 \% \mathrm{~B}$ for $3 \mathrm{~min}$, linear gradient to $100 \% \mathrm{~A}$ in 0.1 min, $100 \%$ A for $0.9 \mathrm{~min}$. The UHPLC was connected to a QEplus Orbitrap mass spectrometer (Thermo Fisher Scientific, Waltham, MA, USA) equipped with heated electrospray ionization source (capillary temperature $360^{\circ} \mathrm{C}$, sheath gas 60 nominal units, aux gas 20 nominal units, sweep gas 5 nominal units, aux gas temperature $400^{\circ} \mathrm{C}$, spray voltage $3.3 \mathrm{kV}$, S-lens RF level $50)$ operated in positive or negative ion mode. Full scan measurements ( $m / z$ 100-1200, resolution 280k, AGC target $3 \cdot 10^{6}$, maxIT $900 \mathrm{~ms}$ ) were performed separately for positive and negative ion mode. $\mathrm{MS}^{2}$ scans with metabolite-specific fragmentation energies were used for metabolite identification.

\section{Isotopologue detection}

Full scan RAW files were converted to mzXML using ProteoWizard msConvert (53) with the vendor's algorithm for peak picking. Isotopologue detection was achieved with R-based $\mathrm{X}^{13} \mathrm{CMS}$ (27). The R script can be found in the Supplementary Information. In brief, after peakpicking with centwave (3 ppp, peakwidth 5-20 s) and retention time alignment with orbiwarp, isotopologues with a mass difference of $1.00335 \mathrm{Da}$ were assigned (RTwindow $10 \mathrm{~s}, 3 \mathrm{ppm}$ ). Either all isotopologue groups $(\alpha=1)$ or only isotopologue groups significantly different from ${ }^{12} \mathrm{C}(\alpha=0.05)$ were reported. Afterwards, significantly increased isotopologue groups were manually curated with reference to the original spectrum in order to exclude groups that did not contain at least 3 consecutive isotopologues.

\section{Compound identification}

Compounds were identified based on their retention time, high resolution mass to charge ratio and fragmentation pattern. Compound Discoverer (Vers. 2.1, Thermo Fisher Scientific, Waltham, MA, USA) was used to predict sum formula, search an in-house and public databases (ChemSpider and mzCloud) as well as calculate FISh scores of candidates. SIRIUS and CSI:FingerID were used to compute fragmentation trees and search molecular structure databases (54). Putatively identified compounds were compared to authentic standards: Arachidonic acid, betaine, carnitine hydrochloride, choline chloride, 11Z-eicosenoic acid, 2hydroxytetradecanoic acid, isoleucine, leucine and 1-oleoyl-sn-glycero-3-phosphocholine were obtained from Sigma Aldrich (Munich, Germany). Oleic acid was purchased from AppliChem (Darmstadt, Germany). Linoleic acid was from Alfa Aesar (Haverhill, MA, USA). 5Z,8Z,11Z,14Z,17Z-Eicosapentaenoic acid was supplied by Cayman Chemicals (Ann Arbor, MI, USA) and 6Z,9Z,12Z-Hexadecatrienoic acid from Larodan (Solna, Sweden). 6Z,9Z,12Z,15- 
Hexadecatetraenoic acid has been synthesized according to Pohnert, Adolph and Wichard (45). ß-Alanine betaine has been synthesized according to Chary, Kumar, Vairamani and Prabhakar (55) Leucine and isoleucine were not baseline separated and are consequently grouped as (iso-)leucine.

\section{Calculation of the degree of labeling}

To calculate the degree of labeling isotopologues are assumed to have a Bernoulli distribution (see Supplementary Information for formula).(56) Measured isotopologue intensities are compared to computed distributions and the squared coefficient of variation between both is minimized in an iterative process.

\section{References}

1. Heesen, W., Planktonkunde - Eine Einführung in die Ökologie der im Wasser schwebenden Kleinwelt. E. Wasserloos, G. Wolff, Eds., MathematischNaturwissenschaftlich-Technische Bücherei (Verlag Otto Salle, Berlin, 1928), vol. 23.

2. Selosse, M. A., Charpin, M., Not, F., Mixotrophy everywhere on land and in water: the grand ecart hypothesis. Ecol. Lett. 20, 246-263 (2017).

3. Stoecker, D. K., Hansen, P. J., Caron, D. A., Mitra, A., Annual, R., "Mixotrophy in the Marine Plankton" Annu. Rev. of Mar. Sci. 9, 311-335 (2017).

4. Flynn, K. J. et al., Misuse of the phytoplanktonzooplankton dichotomy: the need to assign organisms as mixotrophs within plankton functional types. J. Plankton Res. 35, 3-11 (2013).

5. Neilson, A. H., Lewin, R. A., The uptake and utilization of organic carbon by algae: an essay in comparative biochemistry. Phycologia 13, 227-264 (1974).

6. Amblard, C., Carbon Heterotrophic Activity of Microalgae and Cyanobacteria Ecological Significance. Annee Biologique 30, 6-107 (1991).

7. Caron, D. A., Mixotrophy stirs up our understanding of marine food webs. Proc. Natl. Acad. Sci. U.S.A. 113, 2806-2808 (2016).

8. Karlusich, J. J. P., Ibarbalz, F. M., Bowler, C., Phytoplankton in the Tara Ocean. Annu. Rev. Mar. Sci. 12, 233-265 (2020).

9. Mocaer, P. Y., Baudoux, A. C., The essential role of viruses in marine ecology. Virologie 21, 160-172 (2017).

10. Meyer, N., Bigalke, A., Kaulfuss, A., Pohnert, G., Strategies and ecological roles of algicidal bacteria. FEMS Microbiol. Rev. 41, 880-899 (2017). 
11. Borowitzka, M., The physiology of microalgae (Springer Berlin Heidelberg, New York, NY, 2016).

12. Burkholder, J. M., Glibert, P. M., Skelton, H. M., Mixotrophy, a major mode of nutrition for harmful algal species in eutrophic waters. Harmful Algae 8, 77-93 (2008).

13. Ferroni, L., Giovanardi, M., Poggioli, M., Baldisserotto, C., Pancaldi, S., Enhanced photosynthetic linear electron flow in mixotrophic green microalga Ettlia oleoabundans UTEX 1185. Plant Phys. Biochem. 130, 215-223 (2018).

14. Flynn, K. J. et al., Misuse of the phytoplankton-zooplankton dichotomy: the need to assign organisms as mixotrophs within plankton functional types. J. Plankton Res. 35, 3-11 (2013).

15. Raven, J. A., Beardall, J., Flynn, K. J., Maberly, S. C., Phagotrophy in the origins of photosynthesis in eukaryotes and as a complementary mode of nutrition in phototrophs: relation to Darwin's insectivorous plants. J. Exper. Bot. 60, 3975-3987 (2009).

16. Petrou, K., Nielsen, D. A., Uptake of dimethylsulphoniopropionate (DMSP) by the diatom Thalassiosira weissflogii: a model to investigate the cellular function of DMSP. Biogeochemistry 141, 265-271 (2018).

17. Cochlan, W. P., Herndon, J., Kudela, R. M., Inorganic and organic nitrogen uptake by the toxigenic diatom Pseudo-nitzschia australis (Bacillariophyceae). Harmful Algae 8, 111-118 (2008).

18. Vila-Costa, M. et al., Dimethylsulfoniopropionate uptake by marine phytoplankton. Science 314, 652-654 (2006).

19. Mulholland, M. R., Lee, C., Peptide hydrolysis and the uptake of dipeptides by phytoplankton. Limnol. Oceanogr. 54, 856-868 (2009).

20. Hellebust, J. A., Guillard, R. R., Uptake Specificity for Organic Substrates by the Marine Diatom Melosira Nummuloides(1). J. Phycol. 3, 132-136 (1967).

21. Hellebust, J. A., Lewin, J., Transport systems for organic acids induced in the marine pennate diatom, Cylindrotheca fusiformis. Can. J. Microbiol. 18, 225-233 (1972).

22. Lewin, J. C., Lewin, R. A., Auxotrophy and heterotrophy in marine littoral diatoms. Can. J. Microbiol. 6, 127-134 (1960).

23. Spielmeyer, A., Gebser, B., Pohnert, G., Investigations of the uptake of dimethylsulfoniopropionate by phytoplankton. ChemBioChem 12, 2276-2279 (2011). 
24. Paul, C., Pohnert, G., Interactions of the algicidal bacterium Kordia algicida with diatoms: regulated protease excretion for specific algal lysis. PLoS One 6, e21032 (2011).

25. Paul, C., Pohnert, G., Induction of protease release of the resistant diatom Chaetoceros didymus in response to lytic enzymes from an algicidal bacterium. PLoS One 8, e57577 (2013).

26. Bigalke, A., Meyer, N., Papanikolopoulou, L. A., Wiltshire, K. H., Pohnert, G., The Algicidal Bacterium Kordia algicida Shapes a Natural Plankton Community. Appl. Environ. Microbiol. 85 (2019).

27. Huang, X. J. et al., (XCMS)-C-13: Global Tracking of Isotopic Labels in Untargeted Metabolomics. Anal. Chem. 86, 1632-1639 (2014).

28. Pohnert, G., Jung, V., Intracellular compartmentation in the biosynthesis of caulerpenyne: Study on intact macroalgae using stable-isotope-labeled precursors. Org. Lett. 5, 5091-5093 (2003).

29. Bigalke, A., Pohnert, G., Algicidal bacteria trigger contrasting responses in model diatom communities of different composition. MicrobiologyOpen 8 (2019).

30. Sohn, J. H. et al., Kordia algicida gen. nov., sp nov., an algicidal bacterium isolated from red tide. Int. J. System. Evol. Microbiol. 54, 675-680 (2004).

31. Barofsky, A. et al., Growth phase of the diatom Skeletonema marinoi influences the metabolic profile of the cells and the selective feeding of the copepod Calanus spp. J. Plankton Res. 32, 263-272 (2010).

32. Sommer, U. et al., Beyond the Plankton Ecology Group (PEG) Model: Mechanisms Driving Plankton Succession. Annu. Rev. Ecol. Evol. Systemat., 43, 429-448 (2012).

33. Keller, M. D., Kiene, R. P., Matrai, P. A., Bellows, W. K., Production of glycine betaine and dimethylsulfoniopropionate in marine phytoplankton. II. N-limited chemostat cultures. Mar. Bio. 135, 249-257 (1999).

34. Gebser, B., Pohnert, G., Synchronized Regulation of Different Zwitterionic Metabolites in the Osmoadaption of Phytoplankton. Mar. Drugs 11, 2168-2182 (2013).

35. Kageyama, H., Tanaka, Y., Takabe, T., Biosynthetic pathways of glycinebetaine in Thalassiosira pseudonana; functional characterization of enzyme catalyzing three-step methylation of glycine. Plant Physiol. Biochem. 127, 248-255 (2018).

36. Rathinasabapathi, B., Fouad, W. M., Sigua, C. A., beta-Alanine betaine synthesis in the Plumbaginaceae. Purification and characterization of a trifunctional, S-adenosyl-L- 
methionine-dependent N-methyltransferase from Limonium latifolium leaves. Plant Physiol. 126, 1241-1249 (2001).

37. Blunden, G. et al., NMR-Spectra of Betaines from Marine-Algae. Magn. Resonance Chem. 24, 965-971 (1986).

38. Tuchman, N. C., Schollett, M. A., Rier, S. T., Geddes, P., Differential heterotrophic utilization of organic compounds by diatoms and bacteria under light and dark conditions. Hydrobiologia 561, 167-177 (2006).

39. Paik, W. K., Nochumson, S., Kim, S., Carnitine Biosynthesis Via Protein Methylation. Trends Biochem. Sci. 2, 159-161 (1977).

40. Frigeri, L. G., Radabaugh, T. R., Haynes, P. A., Hildebrand, M., Identification of proteins from a cell wall fraction of the diatom Thalassiosira pseudonana - Insights into silica structure formation. Mol. Cell. Proteomics 5, 182-193 (2006).

41. Rettner, J., Werner, M., Meyer, N., Werz, O., Pohnert, G., Survey of the C20 and C22 oxylipin family in marine diatoms. Tetrahedron Lett. 59, 828-831 (2018).

42. Pohnert, G., Boland, W., The oxylipin chemistry of attraction and defense in brown algae and diatoms. Nat. Prod. Rep. 19, 108-122 (2002).

43. Zulu, N. N., Zienkiewicz, K., Vollheyde, K., Feussner, I., Current trends to comprehend lipid metabolism in diatoms. Prog. Lipid Res. 70, 1-16 (2018).

44. D'Ippolito, G. et al., Production of octadienal in the marine diatom Skeletonema costatum. Org Lett 5, 885-887 (2003).

45. Pohnert, G., Adolph, S., Wichard, T., Short synthesis of labeled and unlabeled 6Z,9Z,12Z,15-hexadecatetraenoic acid as metabolic probes for biosynthetic studies on diatoms. Chem. Phys. Lipids 131, 159-166 (2004).

46. Ackman, R. G., Tocher, C. S., McLachla.J, Marine Phytoplankter Fatty Acids. J. Fisheries Res. Board Canada 25, 1603-\& (1968).

47. Dunstan, G. A., Volkman, J. K., Barrett, S. M., Leroi, J. M., Jeffrey, S. W., Essential polyunsaturated fatty-acids from 14 Species of Diatom (Bacillariophyceae). Phytochemistry 35, 155-161 (1994).

48. Chen, Y. C., The biomass and total lipid content and composition of twelve species of marine diatoms cultured under various environments. Food Chem. 131, 211-219 (2012).

49. Indiveri, C. et al., The mitochondrial carnitine/acylcarnitine carrier: Function, structure and physiopathology. Mol. Asp. Med. 32, 223-233 (2011). 
50. Georges, A. A., El-Swais, H., Craig, S. E., Li, W. K. W., Walsh, D. A., Metaproteomic analysis of a winter to spring succession in coastal northwest Atlantic Ocean microbial plankton. ISME J 8, 1301-1313 (2014).

51. Berge, T., Poulsen, L. K., Moldrup, M., Daugbjerg, N., Hansen, P. J., Marine microalgae attack and feed on metazoans. ISME J 6, 1926-1936 (2012).

52. Maier, I., Calenberg, M., Effect of Extracellular Ca2+ and Ca2+-Antagonists on the Movement and Chemoorientation of Male Gametes of Ectocarpus-Siliculosus (Phaeophyceae). Bot. Acta 107, 451-460 (1994).

53. Kessner, D., Chambers, M., Burke, R., Agusand, D., Mallick, P., ProteoWizard: open source software for rapid proteomics tools development. Bioinformatics 24, 25342536 (2008).

54. Duhrkop, K., Shen, H., Meusel, M., Rousu, J., Bocker, S., Searching molecular structure databases with tandem mass spectra using CSI:FingerID. Proc. Natl. Acad. Sci. U.S.A. 10.1073/pnas.1509788112 (2015).

55. Chary, V. N., Kumar, C. D., Vairamani, M., Prabhakar, S., Characterization of amino acid-derived betaines by electrospray ionization tandem mass spectrometry. J. Mass Spectrom. 47, 79-88 (2012).

56. Zachmann, H. G., Mathematik für Chemiker (VCH, Weinheim ; New York, ed. 5., erw. Aufl., 1994), pp. xviii, 700 p.

57. Sumner, L. W. et al., Proposed minimum reporting standards for chemical analysis Chemical Analysis Working Group (CAWG) Metabolomics Standards Initiative (MSI). Metabolomics 3, 211-221 (2007).

\section{Acknowledgments}

We thank W. Kooistra for providing diatom cultures. David Russo is acknowledged for helpful comments on an initial draft of this manuscript.

Funding: Deutsche Forschungsgemeinschaft (DFG) - SFB 1127/2 ChemBioSys - 239748522

Deutsche Forschungsgemeinschaft (DFG) EXC 2051 - Project-ID 390713860. 
643

644

645

646

647

648

649

650

651

652

653

654

655

656

Author contributions: N.M. developed the pipeline and analyzed the data. A.R. acquired and analyzed data. N.M. and G.P. conceived the study, directed all experiments, and wrote the manuscript with contributions from the co-author. All authors approved the manuscript.

Competing interests: Authors declare that they have no competing interests.

Data and materials availability: All mass spectra are deposited in the EMBL metabolomics data repository Metabolights. All computer code (in R) developed for this study is available in the supplementary information.

Supporting Information: This article contains supporting information online at... 


\section{Figures and Tables}

\section{Fig. 1: Labeling of metabolites in Chaetoceros didymus endometabolome after exposure to} metabolites from lysed labeled $S$. costatum. (A) Isotopologue groups were detected and tested for significance using $\mathrm{X}^{13} \mathrm{CMS}$. (B) After manual curation the remaining 548 isotopologue groups were categorized according to their labeling pattern. (C) Isotopologue distributions in metabolites with natural and enriched ${ }^{13} \mathrm{C}$ abundance are depicted for each category. (D) Isotopologue groups were clustered by retention time and $\mathrm{m} / \mathrm{z}$ range. A dot plot showing the correlation between retention time and $\mathrm{m} / \mathrm{z}$ can be found in Suppl. Fig. 2.
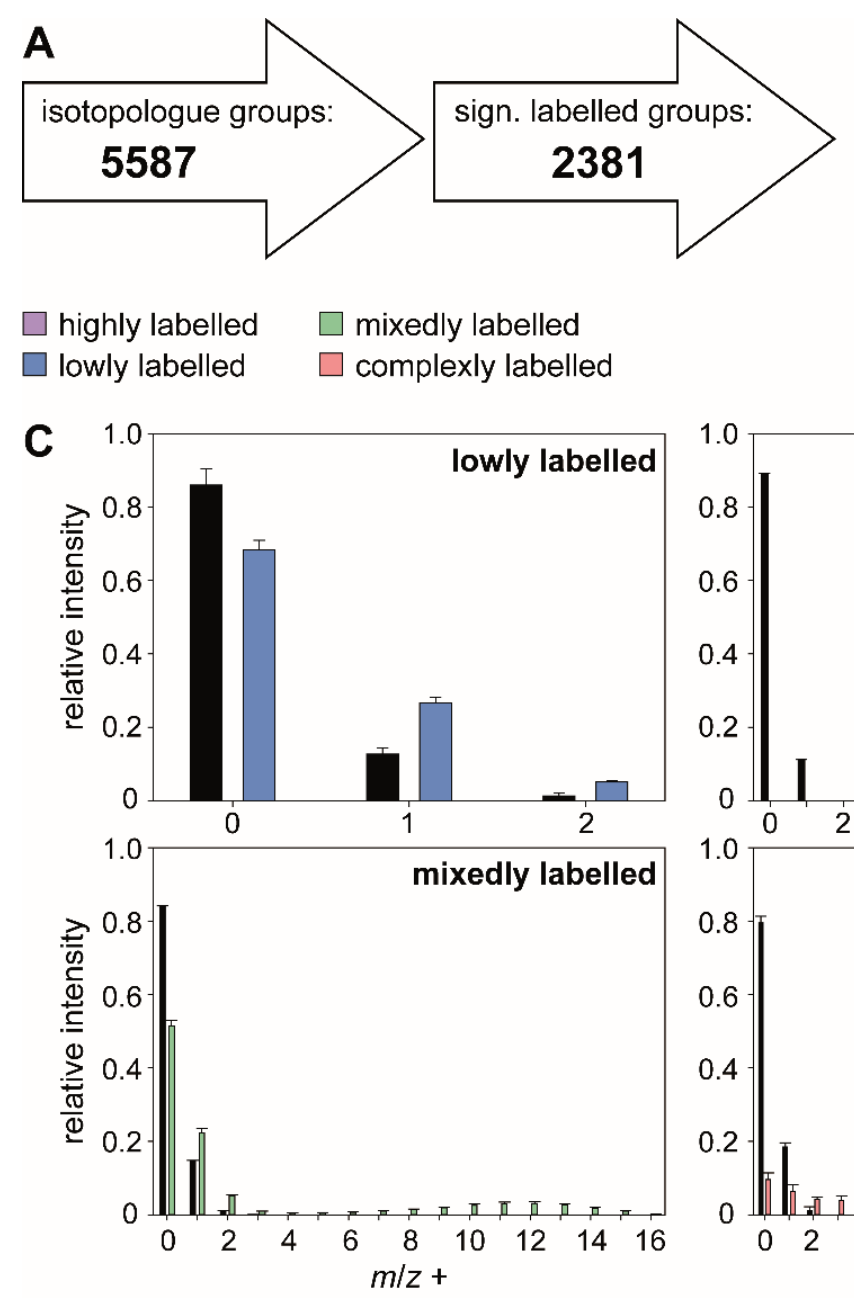

B after manual curation
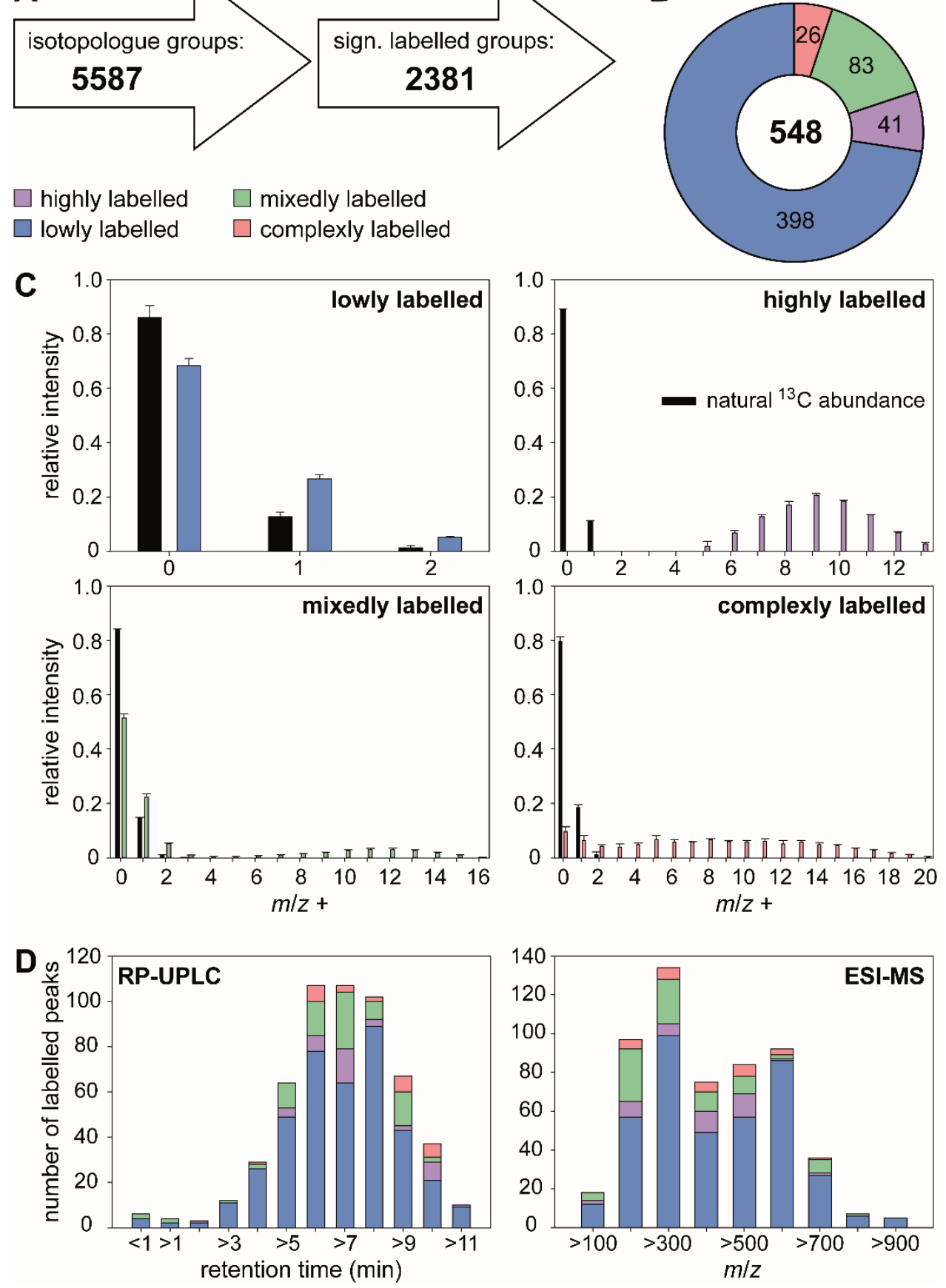
Fig. 2: Labeling pattern of hexadecatetraenoic acid. (A) A mixed labeled metabolite was identified as $6 Z, 9 Z, 12 Z, 15$-hexadecatetraenoic acid. For data evaluation the measured mass spectrum (black bars in D) was divided into a lowly labeled (B) and a highly labeled pool (C). (B) Modelling (grey bars) shows that the lowly labeled pool contains $2.8 \%{ }^{13} \mathrm{C}$ and thus likely derives from anabolism. (C) The highly labeled pool is taken up from the medium, black bars represent measured data of cellular hexadecatetraenoic acid, white bars measured data of hexadecatetraenoic acid in medium. (D) The measured mass spectrum in D can be explained by $20 \%$ hexadecatetraenoic acid derived from uptake and $80 \%$ from de novo synthesis (grey bars). All data are mean \pm SD from biological triplicates.
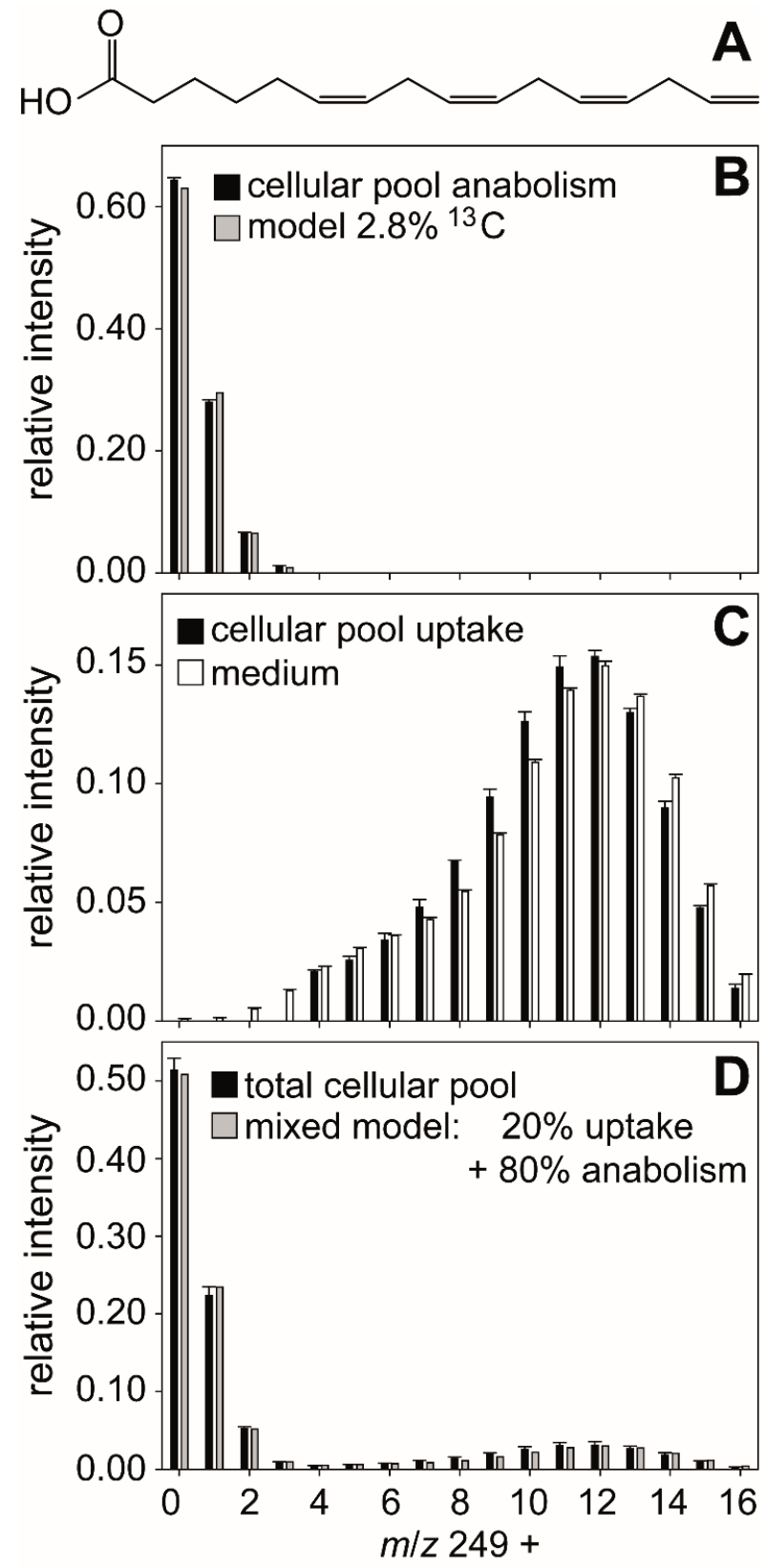
Fig. 3: Complex labeling of lysophosphatidylcholine. All isotopologues (B) of

680 lysophosphatidylcholine (A) are fragmented to yield labeling of the building blocks oleic acid 681 (C) and choline (D). Fragmentation of lysophosphatidylcholine M+8 isotopologue (black bar in $\mathrm{B})$ yields oleic acid (E) with less than four ${ }^{13} \mathrm{C}$, thereby indirectly proving the labeling of glycerol.

684

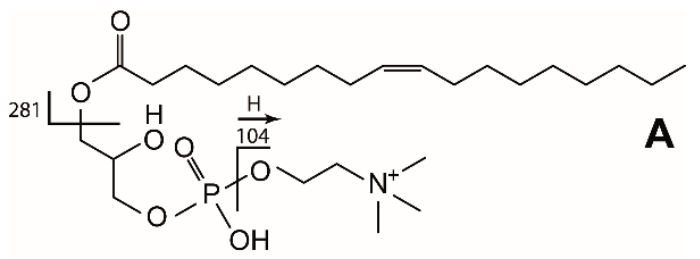
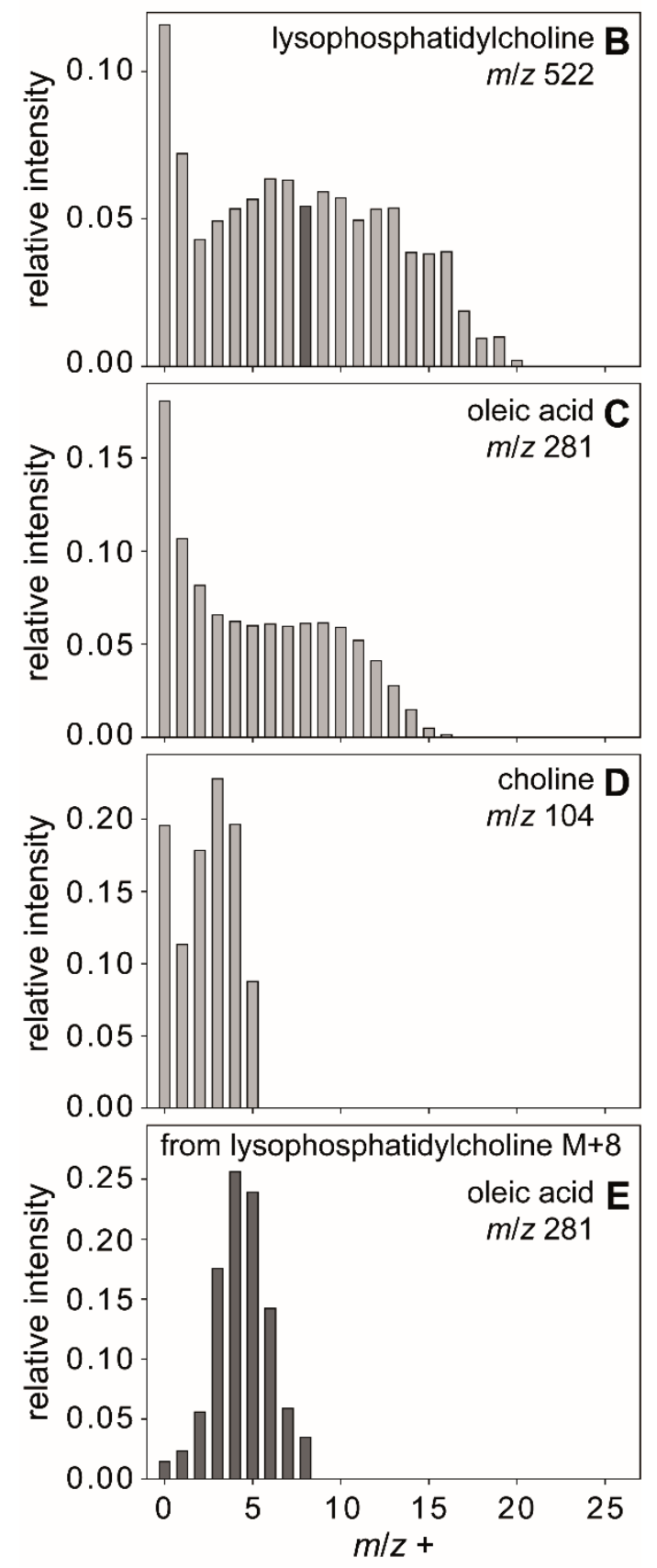
Fig. 4: Uptake and metabolism of labeled organic compounds by Chaetoceros didymus. Organic compounds labeled with ${ }^{13} \mathrm{C}$ (red) are taken up and transformed. Labeled compounds and their catabolic products are mixed with unlabeled metabolites (black) from autotrophic metabolism in anabolic reactions.

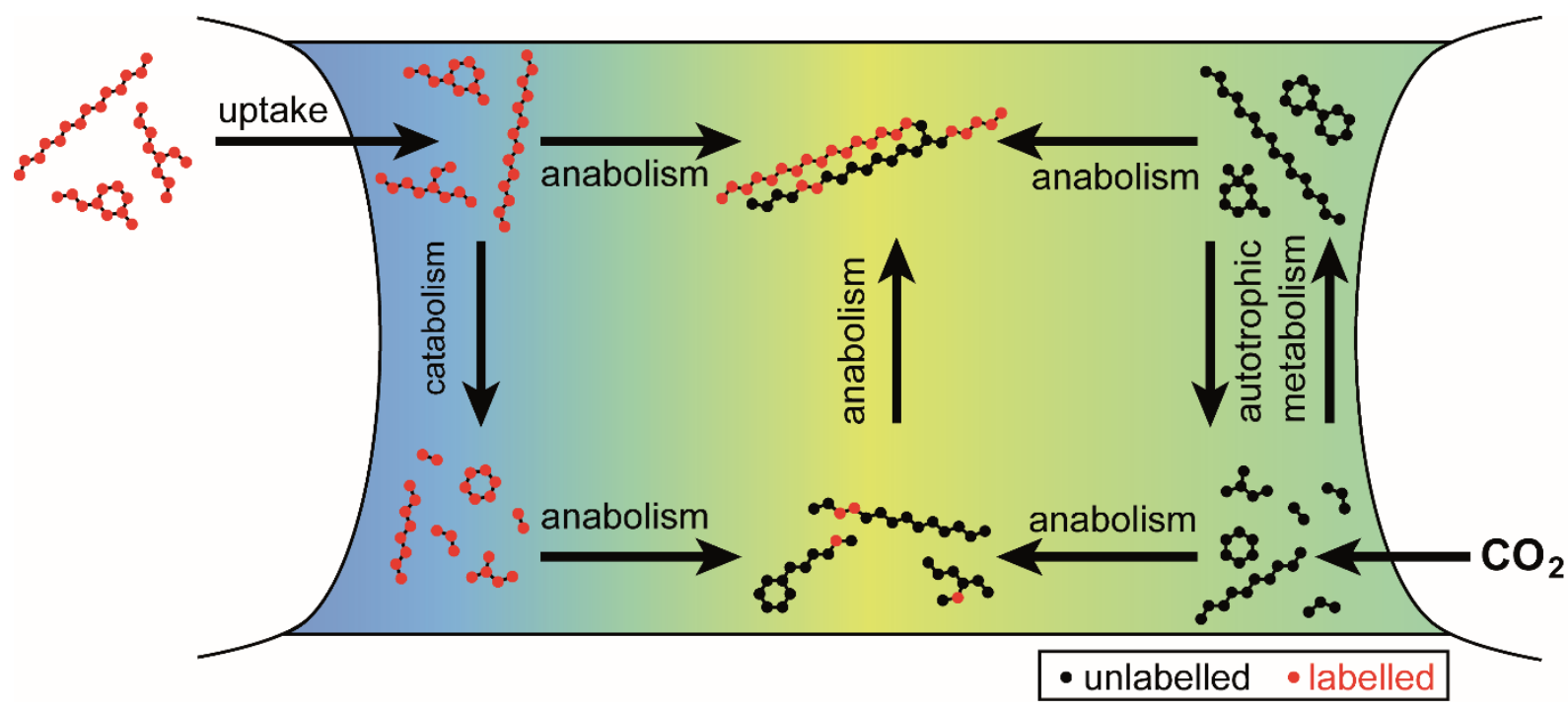




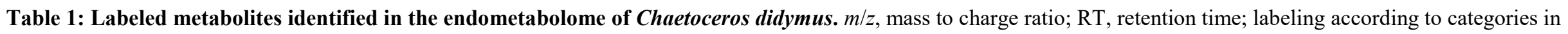
compound class

\begin{tabular}{|c|c|c|c|c|c|c|c|c|}
\hline metabolite & Ion & $m / z$ & $\begin{array}{c}\mathrm{RT} \\
(\min )\end{array}$ & Labeling & Uptake & $\begin{array}{l}\text { Presence in } \\
\text { mixotrophic } \\
\text { medium }\end{array}$ & $\begin{array}{c}\text { lysed by } \\
K \text {. algicida* }\end{array}$ & $\begin{array}{c}\text { Level of } \\
\text { identification }\end{array}$ \\
\hline choline & {$[\mathrm{M}+\mathrm{H}]^{+}$} & 104.1069 & 0.66 & mixed & $15 \%$ & + & - & 1 \\
\hline carnitine & {$[\mathrm{M}+\mathrm{H}]^{+}$} & 162.1125 & 0.66 & mixed & $58 \%$ & - & - & 1 \\
\hline ß-alanine betaine & {$[\mathrm{M}+\mathrm{H}]^{+}$} & 132.1019 & 0.69 & mixed & $53 \%$ & - & - & 1 \\
\hline betaine & {$[\mathrm{M}+\mathrm{H}]^{+}$} & 118.0862 & 0.71 & mixed & $73 \%$ & - & - & 1 \\
\hline (iso-)leucine & {$[\mathrm{M}+\mathrm{H}]^{+}$} & 132.1020 & 1.05 & mixed & $8 \%$ & + & - & 1 \\
\hline $6 Z, 9 Z, 12 Z, 15$-hexadecatetraenoic acid & {$[\mathrm{M}+\mathrm{H}]^{+}$} & 249.1847 & 7.17 & mixed & $20 \%$ & + & + & 1 \\
\hline isomer of 1-oleoyl-sn-glycero-3-phosphocholine & {$[\mathrm{M}+\mathrm{H}]^{+}$} & 522.3554 & 7.25 & complex & $(76 \%)$ & - & - & 3 \\
\hline 1-oleoyl-sn-glycero-3-phosphocholine & {$[\mathrm{M}+\mathrm{H}]^{+}$} & 522.3549 & 7.47 & complex & $(76 \%)$ & - & - & 1 \\
\hline 2-hydroxytetradecanoic acid & {$[\mathrm{M}-\mathrm{H}]^{-}$} & 243.1965 & 7.51 & mixed & $4 \%$ & + & + & 1 \\
\hline $6 Z, 9 Z, 12 Z$-hexadecatrienoic acid & {$[\mathrm{M}+\mathrm{H}]^{+}$} & 251.2004 & 7.59 & mixed & $8 \%$ & + & + & 1 \\
\hline $5 Z, 8 Z, 11 Z, 14 Z, 17 Z$-eicosapentaenoic acid & {$[\mathrm{M}-\mathrm{H}]^{-}$} & 301.2174 & 8.15 & mixed & $3 \%$ & + & + & 1 \\
\hline isomer of arachidonic acid & {$[\mathrm{M}-\mathrm{H}]^{-}$} & 303.2330 & 8.48 & mixed & $6 \%$ & + & - & 3 \\
\hline isomer of linoleic acid & {$[\mathrm{M}-\mathrm{H}]^{-}$} & 279.2329 & 8.88 & mixed & $79 \%$ & + & + & 3 \\
\hline oleic acid & {$[\mathrm{M}-\mathrm{H}]^{-}$} & 281.2486 & 9.39 & complex & $(41 \%)$ & + & + & 1 \\
\hline $11 Z$-eicosenoic acid & {$[\mathrm{M}-\mathrm{H}]^{-}$} & 309.2801 & 10.23 & complex & $(52 \%)$ & - & - & 1 \\
\hline
\end{tabular}


medium preparation

mixotrophic control medium

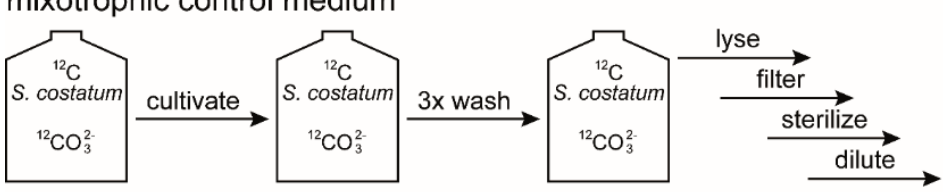

mixotrophic labelled medium

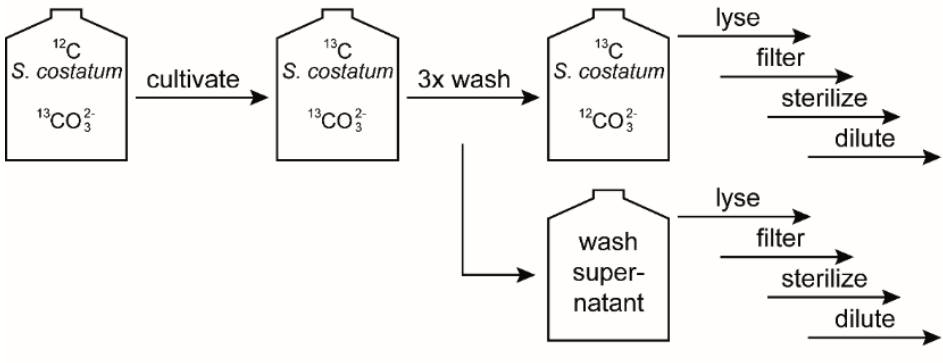

autotrophic medium
703

\section{C. didymus cultivation}

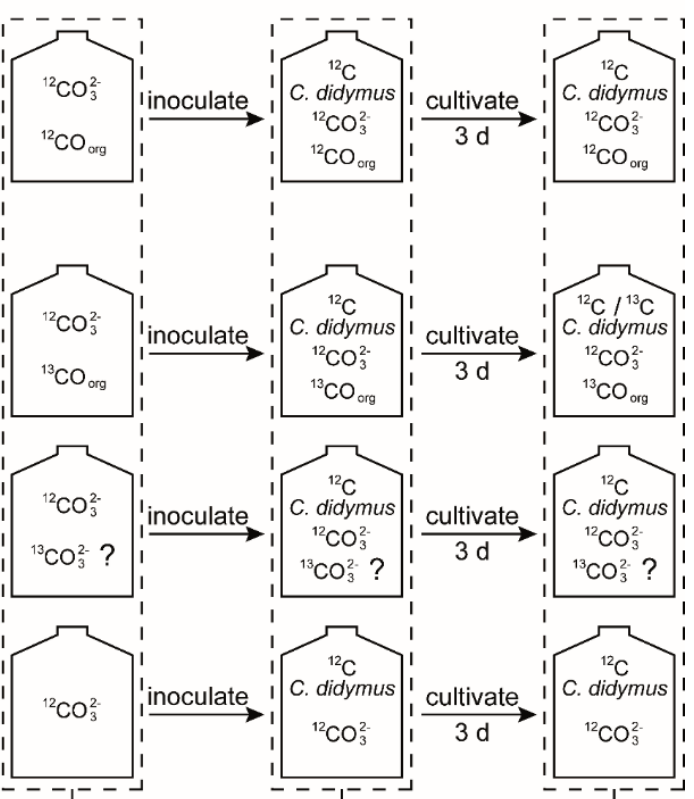

metabolomics ndo-metabolomics exo-metabolomics

endo-metabolomic exo-metabolomics

UHPLC-Orbitrap / $\mathrm{X}^{13} \mathrm{CMS} /$ Compound Discoverer / SIRIUS-CSI:FingerID

Fig. S1. Experimental setup for mixotrophy experiment. Last two lines: To demonstrate the effective removal of inorganic ${ }^{13} \mathrm{C}, \mathrm{C}$. didymus was grown on the wash supernatant and did not contain labelled metabolites 


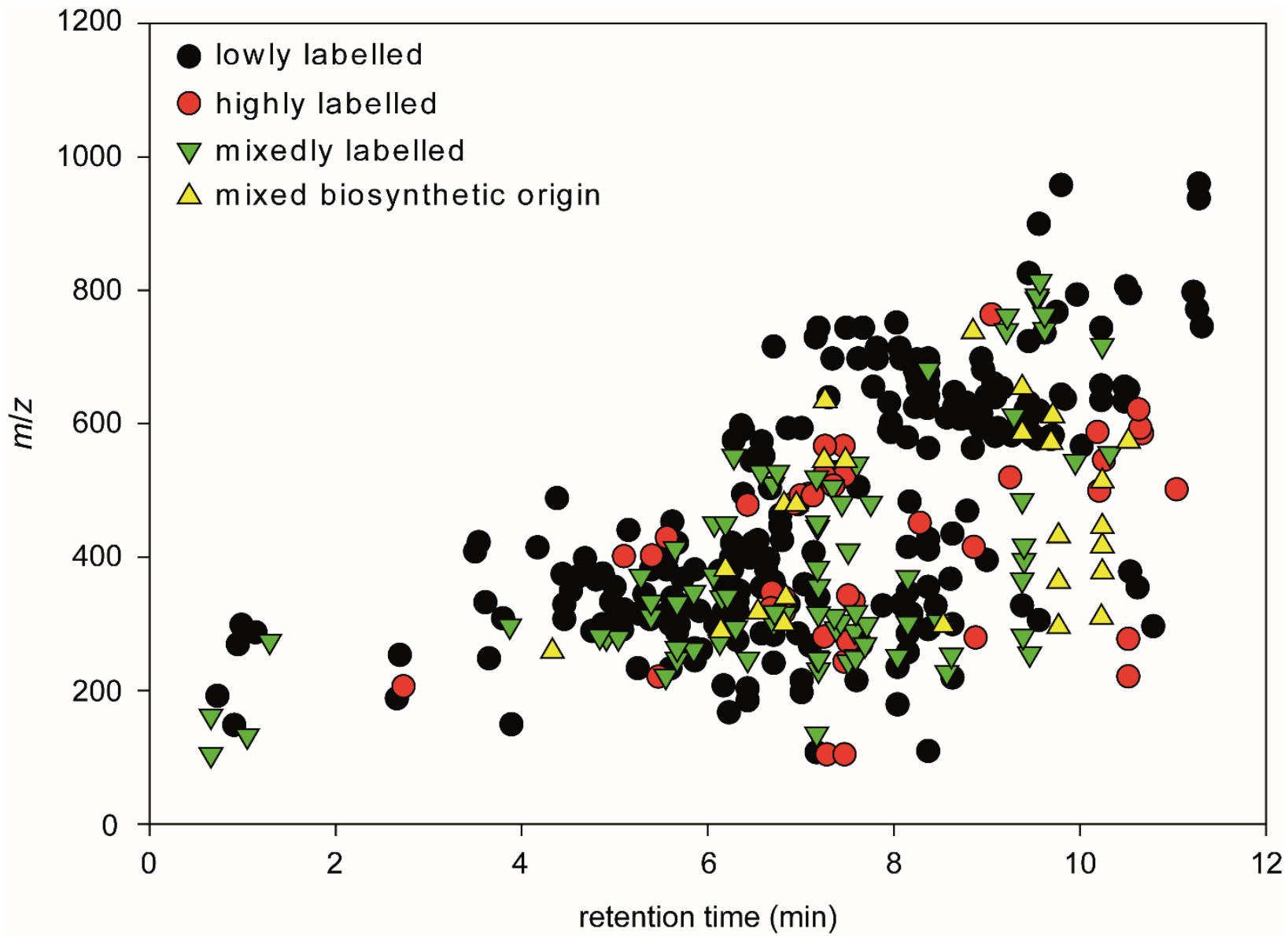

Fig. S2. Labelling of metabolites in Chaetoceros didymus endometabolome. Correlation between retention time (gradient as described in materials and methods) and $\mathrm{m} / \mathrm{z}$ of manually curated isotopologue groups sorted by labelling pattern as described in Fig. 1. 


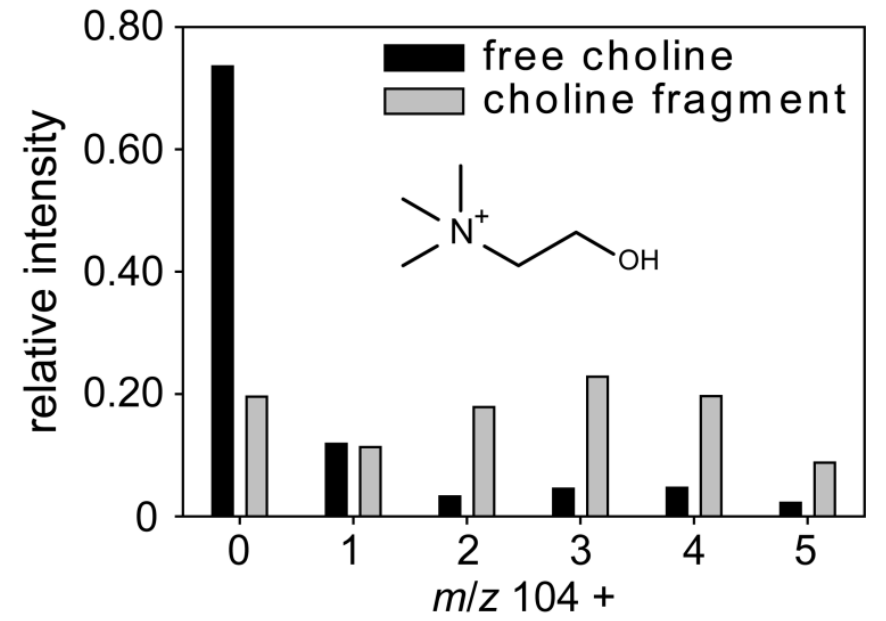

714 Fig. S3. Labelling pattern of carnitine. Depicted are the isotopologues of carnitine. The presence 715 of $\mathrm{M}+5$ to $\mathrm{M}+7$ proves labelled $N$-methyl groups. 
$\underline{\mathrm{R} \text {-script for } \mathrm{X}^{13} \mathrm{CMS} \text { analysis }}$

require(xcms)

require(X13CMS)

\# set working directory to one where the "C12" and "C13" folders reside

setwd("E:/X13CMS")

\# Peak-picking and retention-time alignment with XCMS

$\mathrm{xs}=\mathrm{xcmsSet}(\mathrm{c}(' . / \mathrm{C} 12$ ', './C13'), method= 'centWave', $\mathrm{ppm}=3$, peakwidth $=\mathrm{c}(5,20))$

$\mathrm{xs}=\operatorname{group}(\mathrm{xs}, \mathrm{bw}=5, \mathrm{mzwid}=0.015)$

$\mathrm{xs} 2=\operatorname{retcor}(\mathrm{xs}$, method $=$ 'obiwarp')

$\mathrm{xs} 2=\operatorname{group}(\mathrm{xs} 2, \mathrm{bw}=5, \mathrm{mzwid}=0.025)$

$\mathrm{xs} 3=$ fillPeaks $(\mathrm{xs} 2)$

\# Setting variables for X13CMS

$\mathrm{sN}=$ rownames(xs3@phenoData) \# sample names

$\mathrm{sN}=\mathrm{sN}[\mathrm{c}(1: 3,4: 6)] \#$ samples (3 unlabeled, 3 labeled)

\# -----only significantly different isotopologues ------

\# labeling report for samples:

labelsSign $=$ getIsoLabelReport $(\mathrm{xcmsSet}=\mathrm{xs} 3$, sampleNames $=\mathrm{sN}$, unlabeledSamples $=$ "C12", labeledSamples $=$ "C13", isotopeMassDiff $=1.00335$, RTwindow $=10, \mathrm{ppm}=3$, massOfLabeledAtom $=12$, noiseCutoff $=10000$, intChoice $=$

"intb", varEq = FALSE, alpha $=0.05$, singleSample $=$ FALSE, compareOnlyDistros $=$ FALSE, monotonicityTol $=$ FALSE, enrichTol $=0.1$ )

\# in each of the $\mathrm{sN}$ variables, the first 3 samples listed are of the "C12" or unlabeled type while the next 3 are of the "C13" type

classes $=\mathrm{c}(\mathrm{rep}(" \mathrm{C} 12 ", 3), \operatorname{rep}(" \mathrm{C} 13 ", 3))$

\# print labeling report to a text file (recommended to open in Excel)

printIsoListOutputs(listReport $=$ labelsSign, outputfile = "significant/labels_sign.txt")

\# print pdf of isotopologue groups in a single labeling report plotted as relative intensity distributions

plotLabelReport(isoLabelReport = labelsSign, intOption = "rel", classes, labeledSamples = "C13", outputfile =

"significant/labelsrel_sign.pdf")

\# print pdf of isotopologue groups in a single labeling report plotted as absolute intensity distributions

plotLabelReport(isoLabelReport $=$ labelsSign, intOption = "abs", classes, labeledSamples = "C13", outputfile =

"significant/labelsabs_sign.pdf")

\# -----all isotopologues

\# labeling report for samples:

labelsAll $=$ getIsoLabelReport $(\mathrm{xcmsSet}=\mathrm{xs} 3$, sampleNames $=\mathrm{sN}$, unlabeledSamples $=$ "C12", labeledSamples $=$ "C13", isotopeMassDiff $=1.00335$, RTwindow $=10, \mathrm{ppm}=3$, massOfLabeledAtom $=12$, noiseCutoff $=10000$, intChoice $=$ "intb", varEq = FALSE, alpha = 1, singleSample = FALSE, compareOnlyDistros = FALSE, monotonicityTol = FALSE, enrichTol $=0.1$ )

\# in each of the sN variables, the first 3 samples listed are of the "C12" or unlabeled type while the next 3 are of the "C13" type

classes $=\mathrm{c}\left(\mathrm{rep}\left(" \mathrm{C} 12^{\prime \prime}, 3\right), \operatorname{rep}(" \mathrm{C} 13 ", 3)\right)$

\# print labeling report to a text file (recommended to open in Excel)

printIsoListOutputs(listReport = labelsAll, outputfile = "all/labels_all.txt")

\# print pdf of isotopologue groups in a single labeling report plotted as relative intensity distributions

plotLabelReport(isoLabelReport = labelsAll, intOption = "rel", classes, labeledSamples = "C13", outputfile =

"all/labelsrel_all.pdf")

\# print pdf of isotopologue groups in a single labeling report plotted as absolute intensity distributions plotLabelReport(isoLabelReport $=$ labelsAll, intOption = "abs", classes, labeledSamples = "C13", outputfile = "all/labelsabs_all.pdf")

Bernoulli statistics to calculate the degree of labelling

$I(m)=\frac{n !}{m !(n-m) !} \cdot p^{m} \cdot(1-p)^{n-m}$

For a metabolite with $n$ carbon atoms the intensity of an isotopologue $I(m)$ with $m{ }^{13} \mathrm{C}$ atoms is calculated using the degree of labelling $p$. 